12

\title{
Частотная дисперсия импеданса конденсаторных структур при несимметричном подключении электродов
}

\author{
(C) O.A. Емельянов, И.О. Иванов
}

Санкт-Петербургский политехнический университет Петра Великого, 195251 Санкт-Петербург, Россия

e-mail: oaemel2@gmail.com

(Поступило в Редакцию 5 марта 2017 г. В окончательной редакции 27 июня 2017 г.)

Рассмотрен подход к оценке частотной дисперсии импеданса конденсаторных структур в случае несимметричного противоположного подключения электродов. На основании предложенных уравнений получены точные решения для пространственно-неоднородных распределений потенциала и тока. Полученные решения полностью соответствуют результатам 3D моделирования в программном пакете COMSOL Multiphysics. Частотную дисперсию импеданса необходимо учитывать при проектировании современных электрических конденсаторов для создания эффективных накопителей энергии.

DOI: $10.21883 /$ JTF.2018.01.45493.2238

\section{Введение}

Одним из основных технических показателей электрических конденсаторов является плотность запасаемой энергии $W_{\text {store }}$, определяемая следующим соотношением:

$$
W_{\text {store }}=\frac{C U^{2}}{2 V}
$$

где $C$ - емкость конденсатора с объемом $V, U-$ рабочее напряжение. Электрические конденсаторы с высокой плотностью запасаемой энергии $W_{\text {store }}$ на уровне $0.5 \mathrm{~J} / \mathrm{cm}^{3}$ и выше входят в состав мощных импульсных накопителей энергии, систем лазерной накачки, импульсных модуляторов, дефибрилляторов и т. д. В настоящее время значение $W_{\text {store }}$ импульсных конденсаторов зарубежного производства (Германия, США, Япония) доведено до уровня $1-1.5 \mathrm{~J} / \mathrm{cm}^{3}$ для серийных образцов. Последние тенденции разработок конденсаторной техники развиваются в нескольких направлениях. Одно из них связано с созданием новых типов электродных систем высоковольтных пленочных полимерных конденсаторов, обладающих свойством самовосстановления [1-4]. В соотношении (1) под $C$ часто понимают значение статической емкости $C_{s}$, соответствующей величине приложенного постоянного напряжения. В реальных условиях воздействия переменного (импульсного) напряжения $W_{\text {store }}$ будут определяться эффективной емкостью $C_{\text {eff }}(\omega)<C_{s}$ вследствие потерь энергии в рабочем диэлектрике и металлических частях конденсатора (сопротивление выводов и электродной системы $\left.R_{m e}(\omega)\right)$ и наличием индуктивности $L(\omega)$. При оценке потерь энергии в конденсаторах существенную роль играют диэлектрические потери, связанные с релаксационной поляризацией, обусловливающей частотную дисперсию диэлектрической проницаемости [4]. Частотные зависимости параметров $R_{m e}(\omega)$ и $L(\omega)$ в первую очередь связаны со скин-эффектом и могут быть определены на основе расчетов, детально представленных в [5].
В области достаточно высоких частот (сотни килогерц - мегагерцы) дополнительная частотная дисперсия емкости конденсатора может быть связана с конечными параметрами распространения электромагнитного поля вдоль конденсаторного диэлектрика. Было установлено, что в условиях высокой электродинамической нагрузки происходит сильное снижение эффективной емкости $C_{\text {eff }}$ (в 3 и более раз) при частоте $f>1 \mathrm{kHz}$ [6]. Данное снижение емкости связано с неравномерным распределением потенциала вдоль электродов конденсатора. В этом случае необходимо рассматривать систему электродыдиэлектрик с учетом пространственного распределений тока и напряжения, что приобретает весьма важное значение для расчета конструктивных параметров конденсаторов и подтверждается публикациями последних лет [7-9]. В литературе рассмотрены случаи одностороннего (симметричного) включения электродов конденсаторов относительно приложенного напряжения. Вместе с тем на практике используют двустороннюю схему подключения, анализу которой уделено значительно меньше внимания, и точные расчеты распределения напряжения и тока в конденсаторной структуре в известных литературных источниках не приводятся. Настоящая работа направлена на восполнение указанного пробела и содержит расчет и анализ обсуждаемых полевых характеристик конденсаторов.

\section{Постановка задачи и ее решение}

Рассмотрим две основные схемы подключения электродов конденсатора к источнику напряжения $E$ (рис. 1). Первая схема одностороннего подключения электродов, как правило, используется в конденсаторах с фольговыми электродами $(A)$, а вторая - характерна для металлопленочных и многослойных керамических конденсаторов $(B)$. 
A

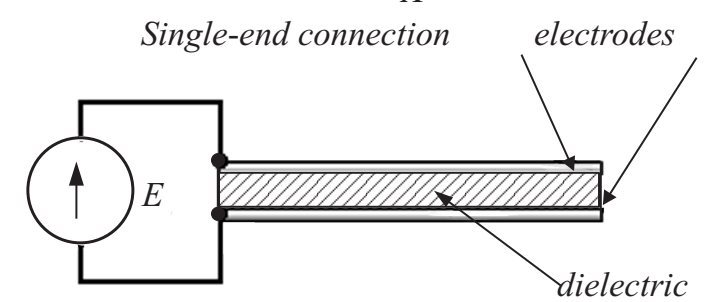

$B$

Double-end connection
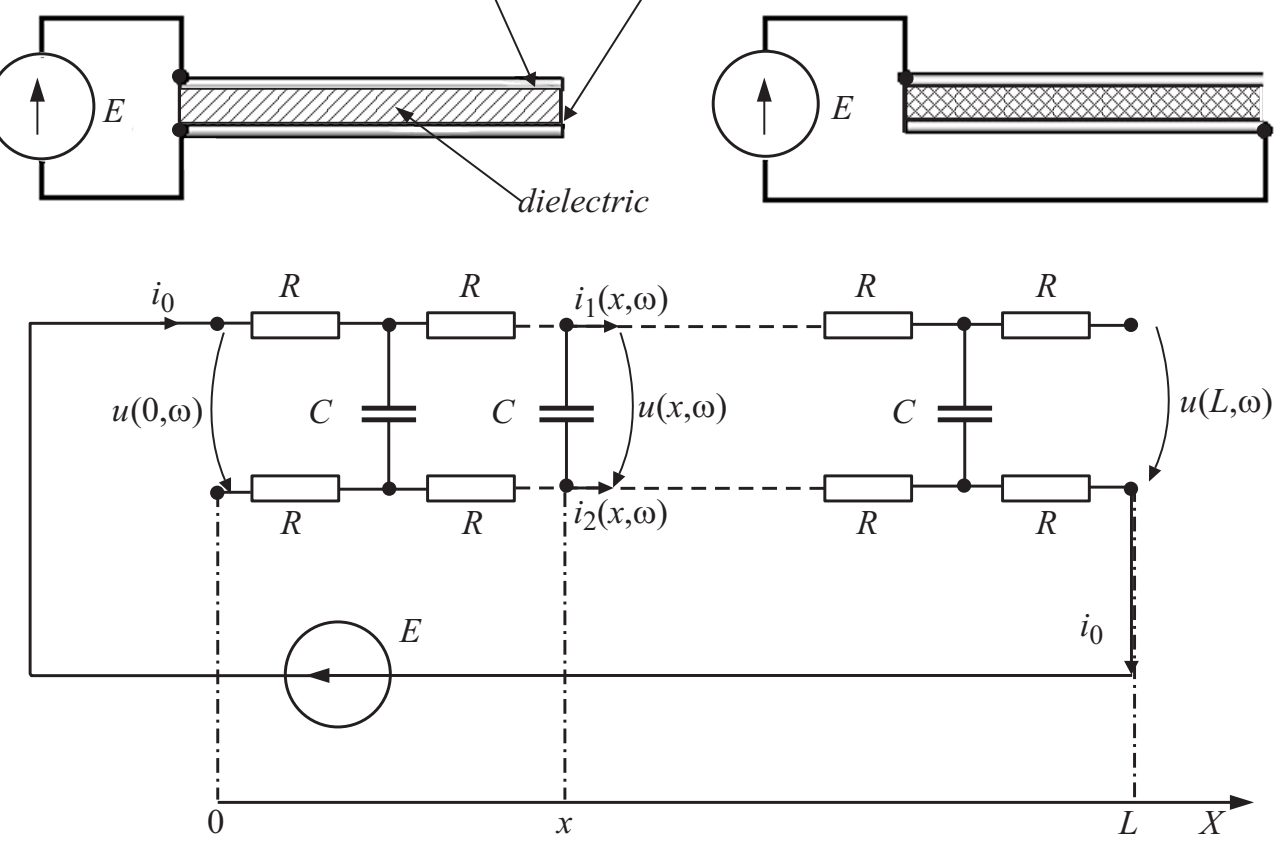

Рис. 1. Два способа подключения электродов конденсаторной структуры и схема замещения для случая $B$.

Обычно для первого случая конденсаторная структура рассматривается в приближении длинной линии с соответствующими погонными параметрами емкости, индуктивности, сопротивления электродов и проводимости диэлектрика. Для реальных случаев обычно погонной индуктивностью и проводимостью можно пренебречь в силу их весьма малых значений, и задача сводится к расчету длинной линии с $R C$ элементами [10-12]. Следует также отметить, что толщины электродов в реальных конденсаторных конструкциях составляют доли микрометров, так что влиянием скин-эффекта при частотах порядка единиц мегагерц можно пренебречь. При анализе первого случая (фольговые электроды) установлено, что пространственное распределение потенциала вдоль электродов практически однородно и его частотная зависимость может наблюдаться в области десятков-сотен мегагерц, что на практике не реализуется, так как эти частоты существенно превышают область работы большинства типов конденсаторов, для которых резонансная частота лежит на уровне долей единиц мегагерц. Вместе с тем вторая схема подключения используется на практике, однако формальный подход использования известных решений телеграфных уравнений для расчета процессов в электродах и диэлектрике в этом случае затруднителен, поскольку источник напряжения включен „по диагонали“ модельной линии. В этом случае в литературе прибегают к численному моделированию процесса или делаются попытки его качественного обоснования на основе классических решений, полученных для первого случая подключения источника напряжения. Общий подход к решению рассматриваемой задачи обсуждался в [13-14] и сводится к теории многополюсников, однако решений рассматриваемой задачи в литературе найти не удалось. Между тем точное решение задачи можно получить, исходя из простых соображений с учетом измененного вида исходных уравнений. Для этого рассмотрим схему замещения конденсаторной структуры в соответствии с рис. 1. Тогда баланс токов и напряжений в произвольной точке $x$ нашей структуры сводится к следующей системе уравнений:

$$
\left\{\begin{array}{l}
-\frac{\partial u(x, t)}{\partial x}=R \Delta i(x, t), \\
-\frac{\partial i_{1}(x, t)}{\partial x}=\frac{\partial i_{2}(x, t)}{\partial x}=C \frac{\partial u(x, t)}{\partial t}, \\
E(t)=u(x, t)+R \int_{0}^{x} i_{1}(x, t) d x+R \int_{x}^{L} i_{2}(x, t) d x,
\end{array}\right.
$$

где $\Delta i(x, t)=i_{1}(x, t)-i_{2}(x, t) ; u(x, t)-$ напряжение между электродами; $i_{1}$ и $i_{2}-$ соответственно токи верхнего и нижнего электродов, $R$ и $C-$ погонные параметры емкости и сопротивления электродов, $E(t)-$ источник напряжения для линии длиной $L$.

Рассмотрим установившийся режим линии в условиях воздействия гармонического источника напряжения $E(t)=E_{m} e^{j \omega t}$, где $E_{m}$ и $\omega-$ амплитуда и частота приложенного напряжения. Тогда для соответствующих 

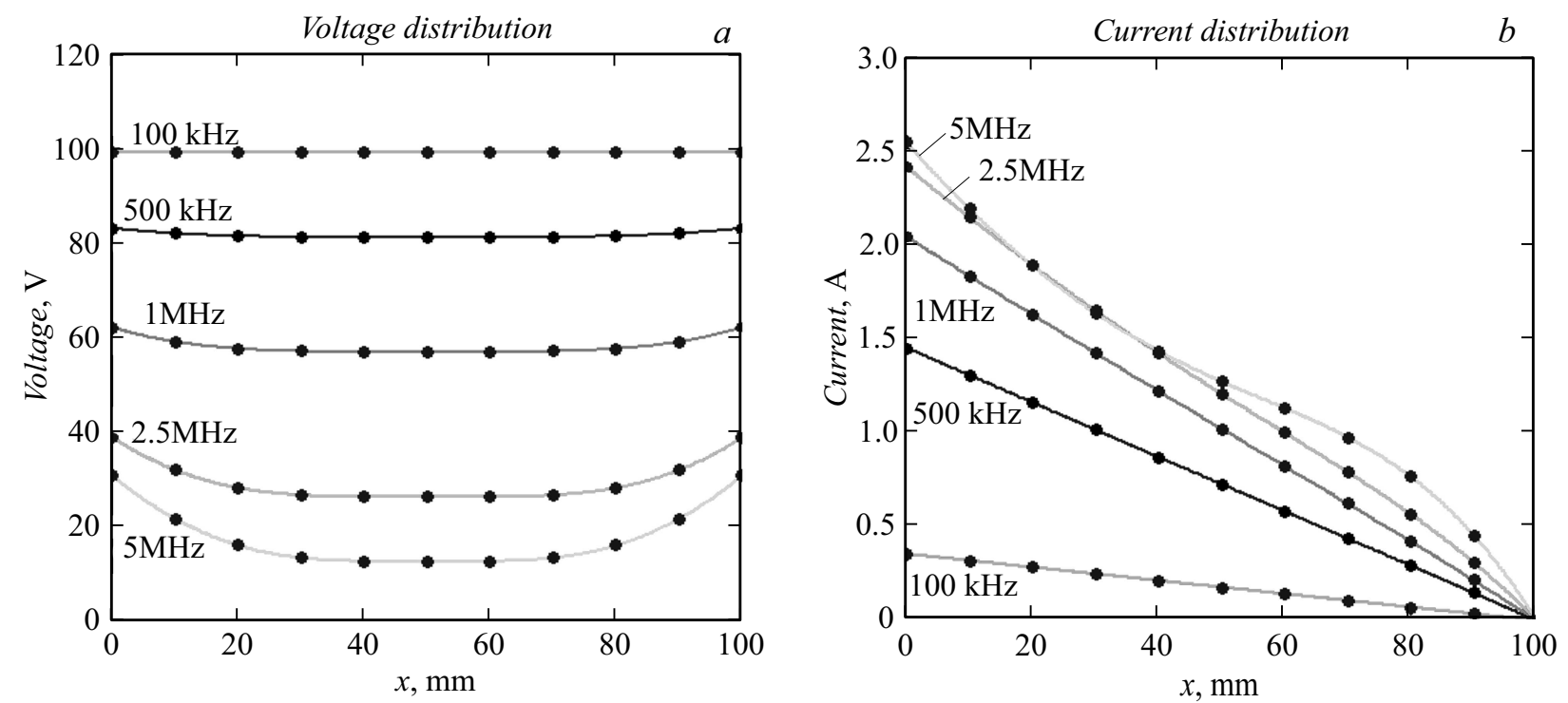

Рис. 2. Пространственное распределение напряжения и тока в конденсаторной структуре при различных частотах. Сплошные линии - моделирование, точки - аналитический расчет.

комплексных амплитуд имеем:

$$
\left\{\begin{array}{l}
-\frac{d \dot{U}(x, \omega)}{d x}=R \Delta \dot{I}(x, \omega), \\
-\frac{d \Delta \dot{I}(x, \omega)}{d x}=2 j \omega C \dot{U}(x, \omega), \\
E_{m}=R \int_{0}^{L} \dot{I}_{1}(x, \omega) d x+\dot{U}(0, \omega) .
\end{array}\right.
$$

Граничные условия соответственно для $I_{0}$ и $U_{0}$ - токов и напряжений в начале и конце линии определяются как

$$
\begin{aligned}
& \dot{I}_{1}(0, \omega)=\dot{I}_{2}(L, \omega)=\dot{I}_{0}, \\
& \dot{I}_{1}(L, \omega)=\dot{I}_{2}(0, \omega)=0, \\
& \dot{U}(0, \omega)=\dot{U}(L, \omega)=\dot{U}_{0} .
\end{aligned}
$$

После несложных выкладок приходим к следующей системе уравнений:

$$
\left\{\begin{array}{l}
-\frac{d \dot{I}_{1}(x, \omega)}{d x}=j \omega C \dot{U}(x, \omega), \\
\frac{d^{2} \dot{U}(x, \omega)}{d x^{2}}-2 j \omega \tau \dot{U}(x, \omega)=0,
\end{array}\right.
$$

где $\tau=R C$.

Решение системы уравнений (5) имеют следующий вид:

$$
\begin{gathered}
\dot{U}(x, \omega)=\frac{\dot{U}_{0} \operatorname{ch}\left(k\left(x-\frac{L}{2}\right)\right)}{\operatorname{ch}\left(\frac{k L}{2}\right)}, \\
\dot{I}_{1}(x, \omega)=\frac{k \dot{U}_{0} \operatorname{ch}\left(\frac{k x}{2}\right)}{R \operatorname{ch}\left(\frac{k L}{2}\right)} \operatorname{sh}\left(\frac{k(L-x)}{2}\right),
\end{gathered}
$$

где

$$
\begin{gathered}
\dot{U}_{0}=\frac{E_{m}}{1+\frac{k l}{2} \operatorname{th}\left(\frac{k l}{2}\right)}, \quad \dot{I}_{0}=\frac{k E_{m} \operatorname{th}\left(\frac{k l}{2}\right)}{R\left[1+\frac{k l}{2} \operatorname{th}\left(\frac{k l}{2}\right)\right]}, \\
k=\sqrt{2 j \omega \tau} .
\end{gathered}
$$

Для верификации полученных решений было проведено соответствующее моделирование в программном пакете COMSOL Multiphysics. В расчетах использовался модуль Electric Currents и стационарный тип решения. Геометрия расчетной модели представляла собой часть конденсаторной секции, выполненной в виде параллелепипеда $(1 \mathrm{~cm} \times 10 \mathrm{~cm} \times 12 \mu \mathrm{m})$. Расчетная модель имела 3 области: полимерную пленку (толщиной $10 \mu \mathrm{m}$ ) и 2 электрода. Для каждой области задавались свои параметры удельной проводимости $\sigma$ и диэлектрической проницаемости $\varepsilon$. Таким образом учитывались погонные параметры: сопротивление $R$ и емкость $C$. При этом произведение $R C=3.4 \cdot 10^{-5} \mathrm{~s} / \mathrm{m}^{2}$, что соответствует значению для реального конденсатора.

Граничными условиями являлись разность потенциалов $E$ на противоположных обкладках (согласно рис. 1) и нулевое значение нормальной составляющей плотности тока $J_{n}=0$ на остальных сторонах расчетной области.

\section{Анализ полученных результатов}

На рис. 2 приведены пространственные распределения напряжения и тока верхнего электрода вдоль конденсаторной структуры в зависимости от частоты приложенного напряжения для модулей аналитических решений (6) и (7) и результатов моделирования. Полученные данные полностью согласуются друг с другом и соответствуют реальным значениям погонной емкости $C=56.6 \mathrm{nF} / \mathrm{m}$ и $R=600 \Omega / \mathrm{m}$ для полимерного конденсаторного диэлектрика толщиной $10 \mu \mathrm{m}$.

Интересно сравнить частотное поведение импеданса и эффективной емкости рассматриваемых схем подключения электродов конденсаторных структур, соответствующих рис. 1 . В этом случае модуль импеданса для схемы $B$ 


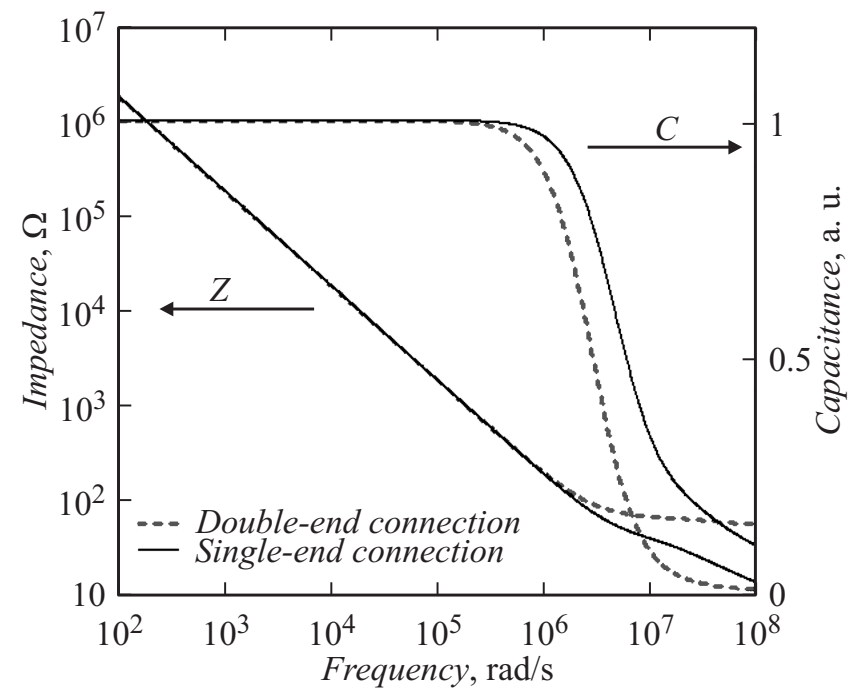

Рис. 3. Частотная дисперсия импеданса и нормированной емкости рассматриваемых конденсаторных структур.

определяется следующим соотношением:

$$
\begin{aligned}
Z_{\text {double }}(\omega) & =\frac{E_{m}}{\left|I_{1}(0, \omega)\right|} \\
& =R L \frac{\sqrt{1+2 h(\omega) \cos (\varphi(\omega))+h^{2}(\omega)}}{2 h(\omega)},
\end{aligned}
$$

где используются вспомогательные параметры

$$
\begin{aligned}
& h(\omega)=\sqrt{\frac{\omega \tau}{2}} L \sqrt{\frac{\operatorname{ch}(\sqrt{\omega \tau} L)-\cos (\sqrt{\omega \tau} L)}{\operatorname{ch}(\sqrt{\omega \tau} L)+\cos (\sqrt{\omega \tau} L)}}, \\
& \cos (\varphi(\omega))=\sqrt{\frac{\operatorname{sh}(\sqrt{\omega \tau} L)-\sin (\sqrt{\omega \tau} L)}{\operatorname{ch}(2 \sqrt{\omega \tau} L)-\cos (2 \sqrt{\omega \tau} L)}} .
\end{aligned}
$$

При этом асимптотика его частотного поведения соответствует конечному пределу

$$
\lim _{\sqrt{\omega \tau} L \rightarrow \infty} Z_{\text {double }}(\omega)=\frac{R L}{2} .
$$

Импеданс схемы $A$ хорошо известен

$$
Z_{\text {single }}(\omega)=\frac{R}{\sqrt{i \omega \tau} \operatorname{th}(\sqrt{i \omega \tau} L)} .
$$

В обоих рассматриваемых случаях эффективная емкость структур и их нормированное значение определяются как

$$
\begin{gathered}
C_{\mathrm{eff}}(\omega)=\operatorname{Re}\left[\frac{1}{i \omega Z(\omega)}\right], \\
C_{N}(\omega)=\frac{C_{\mathrm{eff}}(\omega)}{\lim _{\omega \rightarrow 0} C_{\mathrm{eff}}(\omega)}=\frac{C_{\mathrm{eff}}(\omega)}{L C} .
\end{gathered}
$$

На рис. 3 представлены частотные зависимости импедансов и нормированных емкостей для двух рассматриваемых случаев. Кажущееся слабое различие поведения импедансов (логарифмический масштаб) тем не менее обусловливает более ранний ,завал“ емкости второй структуры на частоте $\sim 200 \mathrm{kHz}$ по отношению к первой, для которой спад начинается при частоте $\sim 1 \mathrm{MHz}$. Таким образом, для реальных параметров пленочных конденсаторов при микросекундных импульсных процессах разряда схема подключения электродов в соответствии с рис. $1(A)$ является более предпочтительной.

\section{Заключение}

На основании предложенных уравнений для противоположного подключения конденсаторных электродов получены точные решения для пространственных распределений потенциала и тока, полностью соответствующие результатам моделирования структуры в программном пакете COMSOL Multiphysics. Неоднородность распределения полевых характеристик приводит к частотным зависимостям импеданса конденсатора и его эффективной емкости. Обсуждаемый подход оценки частотной дисперсии конденсаторных структур позволяет оптимизировать создание и применение эффективных емкостных накопителей энергии. Полученные решения легко обобщаются в случае учета продольной индуктивности и поперечной проводимости и могут быть полезны в теории длинных линий, несимметрично подключаемых к источнику напряжения с противоположных концов.

\section{Список литературы}

[1] Wang H., Blaabjerg F. // IEEE Trans. Ind. Appl. 2014. Vol. 50. N 5. P. 3569-3578.

[2] Jow T.R. et al. // IEEE Pulsed Power Conference. Austin. TX, 2015. P. 1-7.

[3] Belko V.O., Emelyanov O.A. // J. Appl. Phys. 2016. Vol 119. P. 024509.

[4] Бондаренко П.Н., Емельянов О.А. // Письма в ЖТФ. 2005. Т. 31. Вып. 4. С. 67-72.

[5] Шнеерсон Г.А. Поля и переходные процессы в аппаратуре сверхсильных токов. М: Энергоатомиздат, 1992. $413 \mathrm{c.}$

[6] Belko V.O., Emelyanov O.A., Ivanov I.O., Glivenko D.Y. // 2017 IEEE Conf. ElConRus. 2017. P. 1-3.

[7] Qin S., Boggs S. // IEEE Trans. Dielectr. Electr. Insul. 2010. Vol. 17. N 5. P. 1298-1306.

[8] Sivakumar J., Usa S., Panneerselvam M.A. // Res. J. Appl. Sci., Eng. Tech. 2014. Vol. 7. N 3. P. 639-644.

[9] Li Z. et al. // IEEE Trans. Plasma Sci. 2015. Vol. 43. N 6. P. 2038-2045.

[10] Joubert Ch., Béroual A., Rojat G. // J. Appl. Phys. 2004. Vol. 95.N 11. P. 6420-6425.

[11] Brown R.W. // IEEE Trans. Compon. Packag. Manuf. Technol. 2007. Vol. 30. N 4. P. 764-773.

[12] Vuillermet Y. et al. // IEEE Trans. Magn. 2007. Vol. 43. N 4. P. 1569-1572.

[13] Шимони К. Теоретическая электротехника. М.: Мир, 1964. $775 \mathrm{c}$.

[14] Демирчан К.С., Нейман Л.Р., Коровкин Н.В., Чечурин В.Л. Теоретические основы электротехники. СПб.: Питер, 2003. T. $1.576 \mathrm{c}$. 